\title{
An evaluation of the arterial occlusions in peripheral arterial disease by 64-detector multi-slice $\mathrm{CT}$ angiography: DSA correlation
}

\author{
Pelin Seher Oztekin ${ }^{1}$, Alper Sonmez ${ }^{1}$, Fahrettin Kucukay ${ }^{2}$, Derya Oztuna ${ }^{3}$, Umman Sanlıdilek ${ }^{4}$, \\ Ugur Kosar ${ }^{1}$ \\ ${ }^{1}$ Department of Radiology, Ankara Training and Research Hospital, Ankara, Turkey \\ ${ }^{2}$ Department of Radiology, Turkiye Yuksek Ihtisas Hospital, Ankara, Turkey \\ ${ }^{3}$ Department of Biostatistic, Faculty of Medicine, Ankara University, Ankara, Turkey \\ ${ }^{4}$ Department of Radiology, Faculty of Medicine, Ankara University, Ankara, Turkey \\ Email: pelinoztekintr@yahoo.com \\ Received 17 January 2013; revised 17 March 2013; accepted 20 April 2013 \\ Copyright (C) 2013 Pelin Seher Oztekin et al. This is an open access article distributed under the Creative Commons Attribution Li- \\ cense, which permits unrestricted use, distribution, and reproduction in any medium, provided the original work is properly cited.
}

\begin{abstract}
Objective: Evaluation of peripheral arterial disease with 64-detector multi-slice CT angiography (MDCTA) and comparison of the results with the results of digital subtraction angiography (DSA), a standard reference. Materials and Method: The written informed consent of the patients and ethics committee approval were obtained. The retrospective study group consisted of 28 patients with a diagnosis of peripheral arterial disease. Using 64-MDCT-A, the arterial tree of the lower extremity was evaluated for the presence of steno-occlusive lesions that might have led to luminal stenosis. The diagnostic reliability of 64-MDCTA was calculated and compared with that of DSA. Findings: In the segment-based analysis, the sensitivity, specificity, and reliability rates of 64-MDCT angiography in determining significant stenoses were 97.7\%, 97\%, and 97.3\%, respectively. The Kappa coefficiency for compatibility between 64-MDCT-A and DSA methods in grading stenosis was calculated as $0.896(P<0.001)$ and it was statistically significant. Conclusion: 64-MDCT angiography is significantly compatible with DSA method in the evaluation of peripheral arterial diseases. It is a non-invasive method and can be an alternative to DSA.
\end{abstract}

Keywords: Multi-Detector Computer Tomography; Lower Extremity Arteries; Peripheral Arterial Disease; Digital Subtraction Angiography

\section{INTRODUCTION}

Peripheral arterial disease (PAD) is briefly defined as the atherosclerosis of the arteries other than the coronary arteries. The arteries of the lower extremity are the most commonly involved arteries; thus, the arteriosclerotic obstructions of the distal aorta and arteries of the lower extremity are the most common forms of PAD [1]. Nearly 3\% - 4\% of males over 60 years of age also suffer claudication secondary to PAD [2]. In PAD cases, for a proper treatment plan, determination of significant stenoses and occlusion severity as well as correct mapping of the vascular anatomy is of great importance. Digital subtraction angiography (DSA) is the golden standard in the evaluation of PAD. However, it is an invasive method and may lead to morbidity [3-5]. With the advent of multi-sliced CT (MDCT) in the last decade, short scanning time, thin slice, and high spatial resolution were achieved, facilitating the development of 3D reformatted images using the original images in a relatively shorter time [6]. Thereby, it has been possible to image the arterial tree with a single injection of contrast matter. Rubin and colleagues conducted the first study with MDCT angiography in 2001, and the results were reported to be highly compatible with those of DSA [3]. Today, 16- and 64-detector CT technology is used in establishing diagnosis as well as treatment planning.

The clinical use and popularity of 64-MDCT angiography has been increasing, but literature reveals limited number of studies using this imaging method in the evaluation of peripheral arterial diseases of the lower extremity and its correlation with DSA method. Therefore, in this study, entire arterial tree of the patients with peripheral arterial disease, including the distal aorta and distal pedal arteries, was evaluated through 64-MDCT angiography and the correlation of the findings with 
DSA was investigated.

\section{MATERIALS AND METHODS}

We retrospectively evaluated the usefulness of 64MDCT angiography in evaluating peripheral arterial occlusive disease compared with DSA.

\subsection{Patient Population}

Before the study was initiated, all the patients included in the study were informed of the study protocol in detail and their written consents were obtained. The study protocol was also approved by the institutional ethics committee.

Between June 2007 and March 2009, 105 patients were evaluated by MDCT angiography with a prediagnosis of PAD. Of this group, enrolled in the study, 77 were excluded: 42 had declined to undergo DSA on the basis CT angiographic findings, 13 had acute ischemia that required immediate treatment, 10 had impaired renal function (creatinine clearance $<30 \mathrm{ml} / \mathrm{min}$ ), and 12 had not come to our institution after CT angiographic examination. Thus, 28 patients were considered to be candidates for either surgical or radiological intervention and then, they were evaluated by DSA method. Of the patients that comprised the study group $(n=28), 27$ were male and 1 was female. The mean age of the patients was 62.6 years (Range: 41 - 82 years), All the patients were symptomatic. According to Rutherford classification, of the patients, 5 belonged to category $3 ; 9$ belonged to category $4 ; 11$ belonged to category 5 , and 3 belonged to category 6 . Seventeen of the patients with vascular risk had type 2 diabetes mellitus; 23, hypertension, 18, hypercholesterolemia, and 21, a smoking habit. One of the patients had chronic renal failure and was on hemodialysis treatment. The renal function test results of the remaining 27 patients were within normal limits (serum creatine $<1.3 \mathrm{mg} / \mathrm{dl}$ ). Two patients had been amputated at the level of the knee joint of the left lower extremity.

\subsection{4-MDCT Technique}

For the evaluation of the lower extremity arteries, 64detector multi-sliced CT (Toshiba Aquilion, Toshiba Medical systems, Tokyo, Japan) was used. All the patients were placed in supine position and in a neutral position with their feet in the gantry first. The area evaluated was between the suprarenal aorta and the tip of the foot. The scanning parameters were set as follows: tube voltage: $120 \mathrm{kv}$; effective tube flow: $250 \mathrm{mAs}$, rotation time: 0.4 sec, table speed: $29 \mathrm{~mm} / \mathrm{s}$; pitch: $0.844 \mathrm{~mm}$; section thickness: $0.5 \mathrm{~mm}$, and reconstruction interval: $0.5-1$ $\mathrm{mm}$. To achieve optimal contrasting in the vascular structures, automatic bolus tracking was used in the determination of the delay time. At the level of the renal artery orifice, ROI was placed. After the injection of low-dose contrast material, scanning was automatically started when the density in the abdominal aorta reached the level of $180 \mathrm{HU}$. Because the device can only process 1500 sections, the scanning was realized in two stages. In the first stage, the abdominal aorta and above the knee level, and in the second stage, the area between below-the-knee and the tip of the foot were evaluated. For each patient, $130 \mathrm{ml}$ contrast material was used. After infusion of the $20 \mathrm{ml}$ saline solution, the contrast material was infused in biphasic fashion, i.e. $40 \mathrm{ml}$ was given at a rate of $5 \mathrm{ml} / \mathrm{sec}$, and the remaining $90 \mathrm{ml}$, at a rate of $4 \mathrm{ml} / \mathrm{sec}$. After the contrast matter use, $21 \mathrm{cc}$ saline was infused to wash out the veins.

\subsection{DSA}

Catheter angiography was performed using a digital subtraction angiography device (Philips Integris, the Netherlands and Siemens artis FA, Germany). All the patients were evaluated through DSA within 6 weeks ( 1 - 40 days) of MDCT evaluation.

In all the patients, the arterial catheterization was achieved by Seldinger method using a 4-French pigtail catheter. In 26 patients, the catheter was inserted into the superior aortic bifurcation through transfemoral approach, and in 2 patients, because of the presence of Leriche's syndrome (occlusion extending from the aortic bifurcation to the proximal arteries), it was inserted into the ascending aorta through transbrachial approach.

\subsection{Evaluation of the Images and Findings}

The CT images obtained were transferred onto the workstation (vitrea), and the data were processed by using maximum intensity projection (MIP), multiplanar reformatting (MPR), CPR (curved multiplanar), and volume rendering (VR) techniques. All the arterial segments, particularly the axial segments, were studied based on MPR (multiplanar reformat) images. The grading was made using all the algorithms. Two radiologists interpreted the images in the computer medium. MIP and VR reconstructions were formed by removing the bone structures semiautomatically and using the $3 \mathrm{D}$ function of the work-station.

Because of the proximity between the arteries and bone structures at the foot and ankle level, the evaluation was made without the removal of the bone structures.

In the DSA, all the arterial segments, including the distal aorta and proximal foot segments, were imaged in the posteroanterior projection. DSA images were interpreted two radiologists. In the presence of incompatible findings, a consensus was reached. The radiologists were 
blinded to the MDCT findings.

The stenoocclusive lesions in the main arterial segments were graded by CTA and DSA. The maximum number of segments evaluated for each patient was 25 . These segments were the distal abdominal aorta, mainexternal-internal iliac arteries, main-deep-superficial femoral arteries, popliteal arteries, anterior-posterior tibial arteries, peroneal arteries, dorsalis pedis arteries, and plantar arcus.

All the lesions were evaluated using a 5-point scale depending on the stenosis in the lumen diameter. The stenosis in the lumen diameter were measured by visual observations, using a ruler when needed (DSA), and electronic measurements (CTA). The points in the scale represented the following: grade 0 , normal arterial lumen and regular arterial wall; grade 1 , mild stenosis, irregularities in the arterial wall, and less than $50 \%$ stenosis in the arterial diameter; grade 2, moderate stenosis and $50 \%-74 \%$ stenosis in the arterial diameter; grade 3 , severe stenosis and $75 \%$ - $99 \%$ stenosis in the arterial lumen, and grade 4 , occlusion. More than $50 \%$ stenosis ( $\geq$ grade 2) was considered significant. In the presence of multiple lesions within one arterial segment, the stenotic lesion that caused the most significant stenosis was graded.

\subsection{Statistical Analysis}

The statistical analyses were conducted using SPSS 11.5 for Windows (SPSS Incorporation, Chicago, IL, USA).

The diagnostic reliability of CTA in determining significant stenoses (more than $50 \%$, grade $2,3,4$ ) was assessed. DSA results were taken as the standard reference, and for each level, the sensitivity and specificity values were found. To evaluate the compatibility between the two methods in grading the stenotic segment, Cohen kappa co-efficiency (Very good $(\kappa>0.8)$, good $(\kappa$ $=0.61-0.8)$, moderate $(\kappa=0.41-0.6)$, low $(\kappa=0.21$ $0.4)$, very low $(\kappa \leq 0.2))$ was calculated.

\section{RESULTS}

In all the patients, to achieve sufficient and homogenous vascular contrast during the MDCT angiography procedure, automatic scanning method was used with bolus tracking in calculating the delay time. The images obtained from all the patients were of high quality, facilitating reliable evaluation. However, in 2 patients, delayed contrast passage due to widespread grade 4 occlusions lead to false positive results. None of the patients developed venous contamination.

A total of 688 segments were evaluated by contrast CTA and DSA. In all the patients, there was significant stenosis in at least one segment based on the MDCT an- giography and DSA findings. In the CTA, the stenosis in 491 segments were not significant (grade $0-1$ ), while in the DSA, that number was 506. The number of the significant stenotic lesions determined in the $\mathrm{CT}$ (grade 2-3-4) was 197, while in the DSA, it was 182 . The distribution of the number of stenotic segments according to MDCT angiography and DSA findings has been presented in Figure 1. By MDCT angiography, in one patient, main iliac artery aneurysm and in another patient, a patent stent inserted into the femoral artery were detected. The findings for aneurysm and the stent were compatible with those in the DSA.

\subsection{Segment-Based Analysis}

The sensitivity and specificity values of MDCT-A for the lower extremity have been shown in Table 1; the false positive and false negative results, in Table 2, and kappa correlation coefficiency, in Table 3.

\subsection{Aortailiac Arteries}

The findings of MDCT-A and DSA were compatible in $191(97 \%)$ of 196 segments. For the abdominal aorta and main iliac arteries, the findings were fully compatible. In by MDCT-A, a total of 4 nonstenotic segments in the external and internal iliac arteries were evaluated as highgrade, and one significant stenotic segment in the internal iliac arteries was evaluated as low-grade. The sensitivity and specificity of $\mathrm{CT}$ in determining the significant stenoses in the aortailiac arteries were $97 \%$ and $98 \%$ respectively. The compatibility (Kappa value) between the two methods was calculated as 0.910 .

\subsection{Femoropopliteal Arteries}

The MDCT-A and DSA findings were compatible in 217 arteries out of 222. While by CTA, 3 segments with no significant stenoses in the superficial femoral, deep femoral, and popliteal arteries were evaluated as high grade, and one significantly stenotic segment in the su-

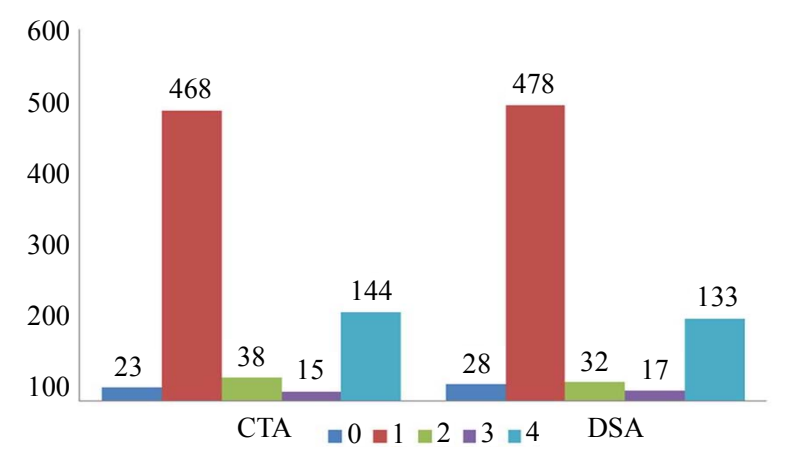

Figure 1. Comparisons of the stenotic segments observed in the CTA and DSA ( 0 , normal arterial lumen; 1 , mild stenosis; 2 , moderate stenosis; 3 , severe stenosis; and 4, occlusion). 
Table 1. Compared to DSA, the diagnostic value of MDCT in detection of the significant stenosis $(>50 \%)$ at the segment level.

\begin{tabular}{lcc}
\hline Location of the lesion & Sensitivity $\%$ & Specificity $\%$ \\
\hline Distal aorta & 100 & 100 \\
Main iliac artery & 100 & 100 \\
External iliac artery & 100 & 97 \\
Internal iliac artery & 90 & 97 \\
Main femoral artery & 96 & 98 \\
Superficial femoral artery & 100 & 99 \\
Deep femoral artery & 100 & 99 \\
Popliteal artery & 100 & 98 \\
Anterior tibial artery & 94 & 98 \\
Posterior tibial artery & 100 & 94 \\
Peroneal artery & 100 & 93 \\
Dorsalis pedis artery & 92 & 94 \\
Plantar arcus & 92 & 97 \\
\hline
\end{tabular}

Table 2. The false positive and false negative results for the segments.

\begin{tabular}{lcc}
\hline Segment & False positive & False negative \\
\hline Distal aorta & 0 & 0 \\
Main iliac artery & 0 & 0 \\
External iliac artery & 2 & 0 \\
Internal iliac artery & 2 & 1 \\
Main femoral artery & 1 & 1 \\
Superficial femoral artery & 0 & 1 \\
Deep femoral artery & 1 & 0 \\
Popliteal artery & 1 & 0 \\
Anterior tibial artery & 1 & 2 \\
Posterior tibial artery & 5 & 0 \\
Peroneal artery & 6 & 0 \\
Dorsalis pedis artery & 5 & 3 \\
Plantar arcus & 1 & 1 \\
Total & 24 & 9 \\
\hline
\end{tabular}

Table 3. The distribution of the Cohen kappa $(\kappa)$ coefficiency values for segments: very high $(\kappa>0.8)$, high $(\kappa=0.61-0.8)$, moderate $(\kappa=0.41-0.6)$, low $(\kappa=0.21-0.4)$, very low $(\kappa \leq$ $0.2)$.

\begin{tabular}{cc}
\hline Segment & Kappa values \\
\hline Distal aorta-iliac & 0.910 \\
Femoropopliteal & 0.940 \\
Tibioperoneal & 0.856 \\
Pedal & 0.813 \\
\hline
\end{tabular}

perficial femoral artery was evaluated as low grade. The sensitivity and specificity of CT in determining the significant stenoses were $99 \%$ and $98 \%$ respectively. The compatibility (Kappa value) between the two methods was calculated as 0.940 .

\subsection{Crural Arteries}

The MDCT-A and DSA findings were compatible in 139 arterial segments out of 162. In the CTA, 12 segments with no significant stenosis (1 segment in the anterior tibial artery; 6 segments in the posterior tibial artery, and 5 segments in the peroneal artery) were evaluated as high grade, while 2 significantly stenotic segments in the anterior tibial artery were evaluated as low grade. The evaluation of luminal opening was highly difficult in the crural arteries with smaller diameters because of widespread wall calcifications, and thus, the number false positive values was higher at this level compared to the those of the other arterial segments. The sensitivity and specificity rates of MDCT-A for the crural arteries were 98\% and 92\% respectively. The compatibility (Kappa value) between the two methods was calculated as 0.856 .

\subsection{Pedal Arteries}

The MDCT-A and DSA findings were compatible for 96 segments out of 106. In the CTA, 6 of the 29 significantly stenotic segments, as determined by DSA, were graded incorrectly as low grade, whereas 4 segments out of 77 with no significant stenosis were graded incurrectly as high grade. The sensitivity and specificity of MDCT-A in determining the significant stenosis at this level were $92 \%$ and $96 \%$. The compatibility (Kappa value) between the two methods was calculated as 0.813 .

In all the segments, CT and DSA were highly compatible in the grading of the stenoses $(\kappa=0.81-1)$.

The samples of CTA and DSA findings are presented in Figures 2-7.

\section{DISCUSSION AND CONCLUSION}

To determine the proper treatment for PAD cases, determination of the location stenotic segments and the occlusion severity in the iliac and lower extremity arteries is required. DSA, known as a gold standard in diagnosis of $\mathrm{PAD}$, is an invasive method; thus, it presents some risks and limitations [3-5]. MDCT-A has been shown to be reliable in the evaluation of occlusion pathologies in the arteries of the lower extremity, and its clinical use has been increasing. With CT techniques before the advent of MDCT, it was not possible to image the entire arterial tree of the lower extremity with only one injection of the contrast material. Because of relatively slower image gain of single-detector CT device and its low z-axis 

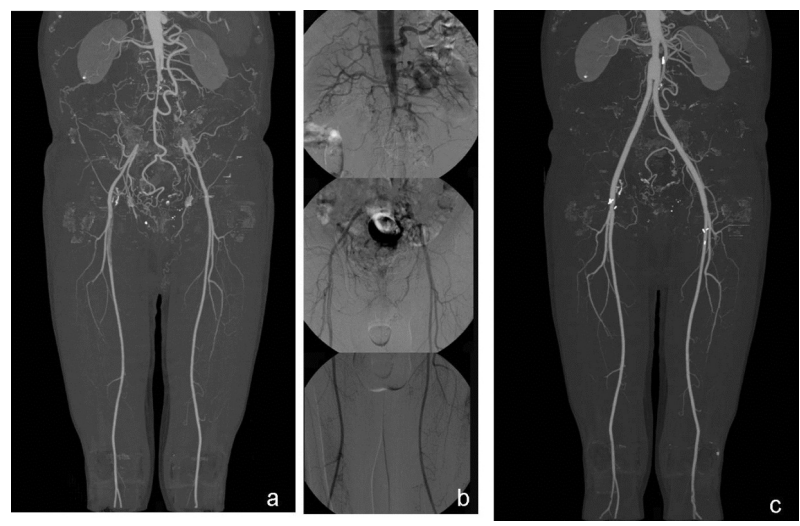

Figure 2. The occlusion (Leriche's disease) in the bilateral main iliac arteries of a 70-year-old man on maximum intensity projection MDCT images (a), as confirmed by DSA (b) is seen. On MDCT MIP images obtained in the postoperative one year, the graft is open (c) (from Oztekin PS peripheral arterial CT angiography Türkiye klinikleri $\mathrm{J}$ radiol-special topics 2012; 5(3):107-14 reprinted by permission).
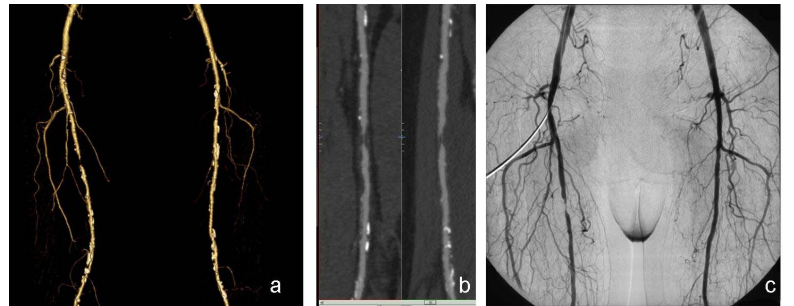

Figure 3. The volume-rendered (VR) images (a), curved multiplanar (CPR) (b), and MDCT images of a 65-year-old male patient show a grade 3 stenosis in the right superficial artery proximal, as confirmed by DSA (c).
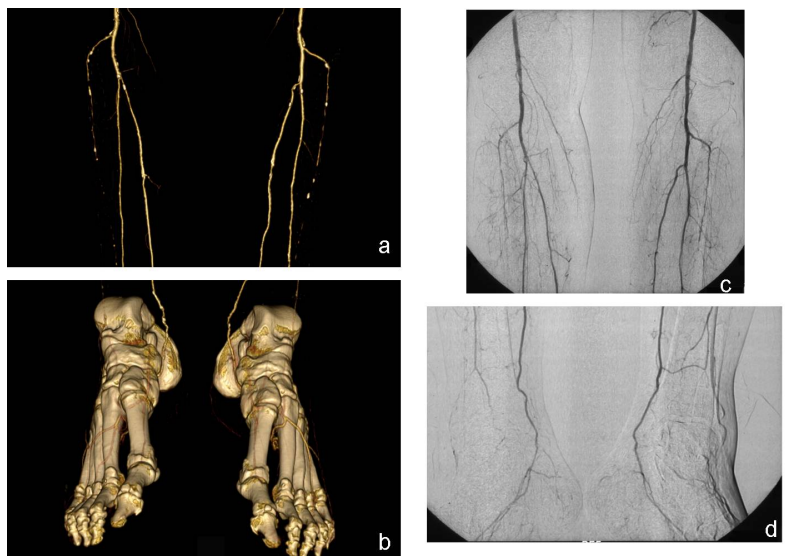

Figure 4. In the VR MDCT images of a 62-year-old male patient obtained at the crural and pedal arteries level, a grade 4 occlusion in the bilateral anterior tibial arteries and a grade 2 stenosis in the left tibialis posterior artery (a) are seen. Bilateral dorsalis pedis and plantar arcuses are open (b). With DSA, the stenosis in the left tibialis posterior artery was evaluated as grade 1(c). All the other findings are similarly evaluated (c,d) (from Oztekin PS Peripheral Arterial CT angiography Türkiye klinikleri J radiol-special topics 2012; 5(3):107-14 reprinted by permission).
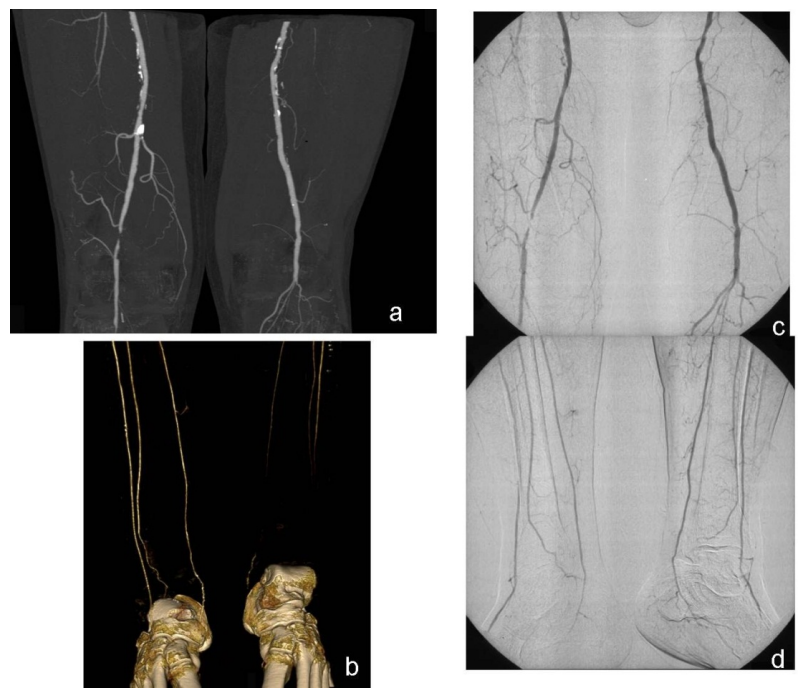

Figure 5. On the maximum intensity projection MDCT images of a 68 -year-old patient, a grade 4 occlusion in a short segment of the distal right femoral artery and a grade 4 occlusion in the left popliteal artery. In the arterial tree of the lower extremity, widespread calcified plaques were noteworthy (a). In the same patient, VR MDCT images at the level of the crural arteries show open flow of the right tibialis anterior-posterior and peroneal arteries and pedal arteries, while on the left, they are occluded (b). DSA results confirmed the occlusions at the level of the right femoral artery and left popliteal artery (c). However, the left crural arteries are open according to DSA results (d).
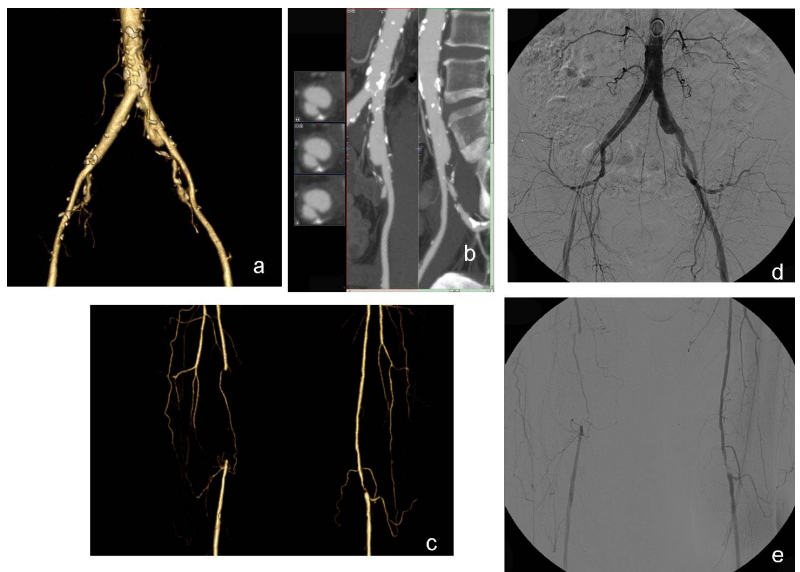

Figure 6. On the VR and CPR MDCT images of a 75-year-old patient (a) and (b), widespread nonstenotic calcifications in the bilateral iliac arteries and saccular aneurysm in the left iliac artery. The VR MDCT images obtained at the femoral level show a grade 4 occlusion on the distal right superficial femoral artery and a grade 4 stenosis in a short segment of the left popliteal artery (c). DSA results were compatible with the results of $\operatorname{MDCT}(\mathrm{d})$ and (e).

resolution, not all the arteries from the renal area to the foot in the lower extremity could be imaged. With the advent of multi-detector CT technique, significant advantages were gained, particularly in scanning time and section thickness, allowing the evaluation of pathologies 


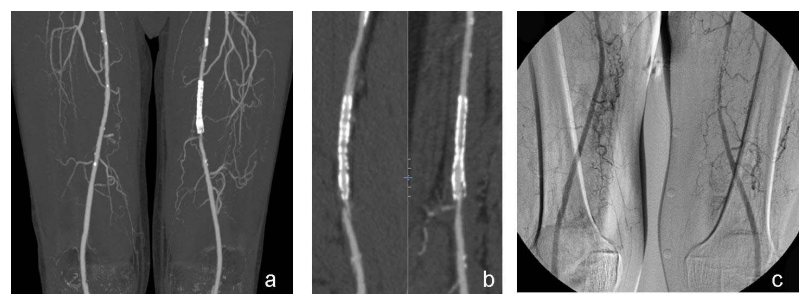

Figure 7. The MIP and CPR MDCT (a) and (b) and control DSA (c) images of a 73-year-old patient show the open stent in the left mid-superficial femoral artery.

in the peripheral arteries.

Literature presents limited number of studies with multi-detector CT on the evaluation of PAD.

In these studies, the sensitivity and specificity of MDCT in determining the stenosis over $50 \%$ were reported to be $90.9 \%-100 \%$ [7-14]. Cernic et al. that compared the 64-MDCT-A results with the results of DSA, a standard reference in the evaluation of stenoocclusive lesions in PAD cases [15]. In that study, the sensitivity and specificity of 64-MDCT in determining the significant stenosis were found to be $97.2 \%$ and $97 \%$, respectively. In 2010, Shareghi et al. and in 2011 Fotiadis et al. studied 28 and 41 patients with haemodynamically significant disease respectively. They found similar results about diagnostic performance of CTA. The sensitivity, specificity, and accurarcy $99 \%, 98 \%$ and $78 \%$ respectively. In our study, these values for 64-MDCT were $97.7 \%$ and $97 \%$, respectively $[16,17]$.

With 64-MDCT, shorter scanning time and less contrast material use are provided, by which sufficient arterial contrast is achieved without a venous circulation. Moreover, 64-MDCT provides thinner sections and higher spatial resolution. However, despite the technical advances, determination of optimal timing for arterial opacification with 64-MDCT device is difficult because the time required for arterial opacification depends on the hemodynamic and vascular state of the patient. Accompanying cardiovascular diseases and any flow obstructions at any point between the infrarenal arteries and pedal arteries or aneurysms will delay the opacification of the arterial tree [5]. Particularly in patients with unilateral severe stenosis, asymmetric contrasting may develop [18]. In the same vein, despite the correct calculation of delay time in 64-MDCT, insufficient opacification may occur in the distal arteries because of rapid scanning.

In our study, the scanning was realized by determination of the delay time through bolus timing method. The images of all the arterial segments, including the pedal arteries, were of high quality in all the patients including the complex patients (those with Lerisch syndrome and widespread occlusions). None of the patients developed venous contamination. Nevertheless, in two patients with severe unilateral stenooclusive lesions, delayed contrast material passage resulted in false positive findings.

Laswed et al. conducted the only detailed study that focused on the pedal arteries, and reported sensitivity and specificity rates of $91 \%$ and $96 \%$ for MDCT in evaluations of crural and pedal arteries [5]. In our study, the sensitivity and specificity rates of MDCT at these levels were $94 \%$ and $96 \%$. In our study, because no contrast enhancement was observed in the plantar arcs of one patient in the DSA evaluation, the segment under evaluation was considered to have occlusion. However, this particular segment was imaged as intact on MDCT, but based on the DSA results, regarded as golden standard, the result was false negative. In our study, the segments where there was lack of compatibility were evaluated as either one grade higher or one grade lower. Semiquantitative nature of evaluation led to differences in grading, particularly for stenotic lesions at threshold values. Moreover, while CT images could be reconstructed in $3 \mathrm{D}$ format, DSA yielded only 2D images, and evaluation of a single image obtained in posteroanterior projection caused differences in the results.

Another disadvantage of CTA is the challenges experienced at times in the evaluation of severely calcified arteries. Ota et al. have emphasized that in the presence of severe calcification, grading of the stenosis is more difficult [2]. On the other hand, 64-MDCT yields images with thinner sections and higher spatial resolution, and thus, this problem is avoided. In the present study, the characteristics of the plaque structure and their area of involvement could not be evaluated in detail. However, in a great majority of our patients, there were severe arterial calcifications. With careful evaluations of the multiplanar images, no problems were encountered in the assessment of stenosis at the aortailiac and femoropoplietal levels. In the evaluation of crural arteries with thin calibers, however, severely calcified plaques led to false positive results. The statistical evaluation showed significant compatibility between MDCT and DSA results.

Portugaller et al reported that when volume rendering technique was used alone, the sensitivity and specificity rates in detecting the high-grade stenosis $(>75 \%)$ were $84 \%$ and $78.5 \%$ respectively. With the use of MIP, however, the sensitivity and specificity rates were $89 \%$ and $74 \%$. In the same study, the best results were obtained by evaluation of the axial images and the sensitivity and specificity rates were found to be $92 \%$ and $83 \%$ [11]. Schernthaner et al. used 16-detector CT as reconstruction algorithm in MIP and multipath CPR techniques and found sensitivity and specificity rates of $99.2 \%$ and $100 \%$ [8]. In our study, to minimize the data loss associated with threshold values selected in the $3 \mathrm{D}$ techniques, the MPR axial images of all the segments were carefully studied and 3D reconstructions were formed and evalu- 
ated. Although no statistical analysis of these data was conducted for the evaluation time, it was observed that MDCT evaluation took longer than DSA evaluations (mean time for MDCT: 35 minutes, mean time for DSA: 15 minutes).

In conclusion, 64-MDCT-A is successful in the imaging of peripheral arterial disease of the entire arterial tree of the lower extremity, including the distal pedal arteries, and it can be considered an noninvasive alternative to DSA.

\section{REFERENCES}

[1] Garcia, L.A. (2006) Epidemiology and pathophysiology of lower extremity peripheral arterial disease. Journal of Endovascular Therapy, 13, 113-119.

doi:10.1583/05-1751.1

[2] Ota, H., Takase, K., Rikimaru, H., Tsuboi, M., Yamada, T., Sato, A., et al. (2005) Quantitative vascular measurements in arterial occlusive disease. RadioGraphics, 25, 1141-1158. doi:10.1148/rg.255055014

[3] Rubin, G.D., Schmidt, A.J., Logan, L.J. and Sofilos, M.C. (2001) Multidetector row CT angiography of lower extremity arterial inflow and runoff. Initial experience. $R a$ diology, 221, 146-158. doi:10.1148/radiol.2211001325

[4] Reimer, P. and Landwehr, P. (1998) Non-invasive vascular imaging of peripheral vessels. European Radiology, 8, 858-872. doi: $10.1007 / \mathrm{s} 003300050483$

[5] Laswed, T., Rizzo, E., Guntern, D., Doenz, F., Denys, A., Schnyder, P., et al. (2008) Assessment of occlusive arterial disease of abdominal aorta and lower extremities arteries: Value of multidetector CT angiography using an adaptive acquisition method. European Radiology, 18, 263-272. doi:10.1007/s00330-007-0749-0

[6] Hiatt, M.D., Fleischmann, D., Hellinger, J.C. and Rubin, G.D. (2005) Angiographic imaging of the lower extremities with multidetector CT. Radiologic Clinics of North America, 43, 1119-1127. doi:10.1016/j.rcl.2005.08.008

[7] Ofer, A., Nitecki, S.S., Linn, S., Epelman, M., Fischer, D., Karram, T., et al. (2003) Multidetector CT angiography of peripheral vascular disease: A prospective comparison with digital subtraction angiography. American Journal of Roentgenology, 180, 719-724. doi:10.2214/ajr.180.3.1800719

[8] Schernthaner, R., Stadler, A., Lomoschitz, F., Weber, M., Fleischmann, D., Lammer, J., et al. (2008) Multidetector CT angiography in the assessment of peripheral arterial occlusive disease: Accuracy in detecting the severity, number, and length of stenoses. European Radiology, 18, 665671. doi:10.1007/s00330-007-0822-8

[9] Catalano, C., Fraioli, F., Laghi, A., Napoli, A., Bezzi, M.,
Pediconi, F., et al. (2004) Infrarenal aortic and lower-extremity arterial disease: Diagnostic performance of multidetector row CT angiography. Radiology, 231, 555-563. doi:10.1148/radiol.2312020920

[10] Martin, M.L., Tay, K.H., Flak, B., Fry, P.D., Doyle, D.L., Taylor, D.C. et al. (2003) Multidetector CT angiography of the arterial system and lower extremities: A prospective comparison with digital subtraction angiography. American Journal of Roentgenology, 180, 1085-1091. doi:10.2214/ajr.180.4.1801085

[11] Portugaller, H.R., Schoellnast, H., Hausegger, K.A., Tiesenhausen, K., Amann, W. and Berghold, A. (2004) Multislice spiral CT angiography in peripheral arterial occlusive disease: A valuable tool in detecting significant arterial lumen narrowing? European Radiology, 14, 16811687. doi:10.1007/s00330-004-2289-1

[12] Willmann, J.K., Baumert, B., Schertler, T., Wildermuth, S., Pfammatter, T., Verdun, F.R., et al. (2005) Aortoiliac and lower extremity arteries with 16-detector row CT angiography: Prospective comparison with digital subtraction angiography. Radiology, 236, 1083-1093. doi:10.1148/radiol.2362040895

[13] Albrecht, T., Foert, E., Holtkamp, R., Kirchin, M.A., Ribbe, C., Wacker, F.K., et al. (2007) 16-MDCT angiography aortoiliac and lower extremity arteries: Comparison with digital subtraction angiography. American Journal of Roentgenology, 189, 702-711. doi:10.2214/AJR.07.2333

[14] Ota, H., Takase, K., Igarashi, K., Chiba, Y., Haga, K., Saito, H., et al. (2004) MDCT compared with digital subtraction angiography for assessment of lower extremity arterial occlusive disease: Importance of reviewing crosssectional images. American Journal of Roentgenology, 182, 201-209. doi:10.2214/ajr.182.1.1820201

[15] Cernic, S., Mucelli, F.P., Pellegrin, A., Pizzolato, R. and Cova, M.A. (2009) Comparison between 64-row CT angiography and digital subtraction angiography in the lower extremities: Personal experience. La Radiologia Medica, 114, 1115-1129. doi:10.1007/s11547-009-0457-8

[16] Shareghi, S., Gopal, A., Gul, K., Matchinson, J.C., Wong, C.B., Weinberg, N., et al. (2010) Diagnostic accuracy of 64 mutidedector computed tomographic angiography in peripheral vascular disease. Catheterization and Cardiovascular Interventions, 75, 23-31.

[17] Fotiadis, N., Kyriakides, C., Bent, C., Vorvolakos, T. and Matson, M. (2011) 64-section CT angiography in patients with critical limb ischaemia and severe claudication: Comparison with digital subtractive angiography. Clinical Radiology, 66, 945-952. doi:10.1016/j.crad.2011.04.015

[18] Kock, M.C., Dijkshoorn, M.L., Pattynama, P.M. and Myriam Hunink, M.G. (2007) Multi-detector row computed tomography of peripheral arterial disease. European $R a-$ diology, 17, 3208-3222. doi:10.1007/s00330-007-0729-4 